\title{
Biideals in BCK/BCI-Bialgebras
}

\author{
YOUNG BAE JuN \\ Department of Mathematics Education (and RINS), Gyeongsang National Univer- \\ sity, Chinju 660-701, Korea \\ e-mail : skywine@gmail.com
}

Abstract. The biideal structure in BCK/BCI-bialgebras is discussed. Relationships between sub-bialgebras, biideals and IC-ideals (and/or CI-ideals) are considered. Conditions for a biideal to be a sub-bialgebra are provided, and conditions for a subset to be a biideal (resp. IC-ideal, CI-ideal) are given.

\section{Introduction}

A BCK/BCI-algebra is an important class of logical algebras introduced by $\mathrm{K}$. Iséki and was extensively investigated by several researchers. Bialgebraic structures, for example, bisemigroups, bigroups, bigroupoids, biloops, birings, bisemirings, binear-rings, etc., are discussed in [4]. In [2], Jun et al. established the structure of BCK/BCI-bialgebras, and investigated some properties. In this paper, we introduce the notion of biideals, IC-ideals and/or CI-ideals in BCK/BCIbialgebras. We discuss relationships between biideals, IC-ideals (and/or CI-ideals) and sub-bialgebras, and give conditions for a biideal to be a sub-bialgebra. We also provide conditions for a subset to be a biideal (resp. IC-ideal, CI-ideal).

\section{Preliminaries}

An algebra $(X ; *, 0)$ of type $(2,0)$ is called a $B C I$-algebra if it satisfies the following conditions:

(I) $(\forall x, y, z \in X)(((x * y) *(x * z)) *(z * y)=0)$,

(II) $(\forall x, y \in X)((x *(x * y)) * y=0)$,

(III) $(\forall x \in X)(x * x=0)$,

(IV) $(\forall x, y \in X)(x * y=0, y * x=0 \Rightarrow x=y)$.

If a BCI-algebra $X$ satisfies the following identity:

(V) $(\forall x \in X)(0 * x=0)$,

Received April 28, 2006, and, in revised form, June 15, 2008.

2000 Mathematics Subject Classification: 06F35, 03G25.

Key words and phrases: BCK/BCI-bialgebras, sub-bialgebras, biideals. IC-ideal, CIideal. 
then $X$ is called a $B C K$-algebra. A nonempty subset $S$ of a BCK/BCI-algebra $X$ is called a subalgebra of $X$ if $x * y \in S$ for all $x, y \in S$. A subset $H$ of a BCK/BCIalgebra $X$ is called an ideal of $X$, written by $H \triangleleft X$, if it satisfies the following axioms:

- $0 \in H$,

- $(\forall x \in X)(\forall y \in H)(x * y \in H \Rightarrow x \in H)$.

Any ideal $H$ of a BCK/BCI-algebra $X$ satisfies the following implication:

$$
(\forall x \in X)(\forall y \in H)(x \leq y \Rightarrow x \in H) .
$$

A subset $A$ of a BCI-algebra $X$ is called a closed ideal of $X$, denoted by $A \triangleleft_{c} X$, if it is an ideal of $X$ such that $0 * x \in A$ for all $x \in A$. We refer the reader to the book [3] for further information regarding BCK/BCI-algebras.

\section{Biideals of BCK/BCI-bialgebras}

Definition 3.1 ([2]). Let $X=(X, *, \oplus, 0)$ be an algebra of type $(2,2,0)$. Then $X=(X, *, \oplus, 0)$ is called a $B C K$-bialgebra (resp. BCI-bialgebra) if there exists two distinct proper subsets $X_{1}$ and $X_{2}$ of $X$ such that

(i) $X=X_{1} \cup X_{2}$.

(ii) $\left(X_{1}, *, 0\right)$ is a BCK-algebra (resp. BCI-algebra).

(iii) $\left(X_{2}, \oplus, 0\right)$ is a BCK-algebra (resp. BCI-algebra).

Denote by $X=K\left(X_{1}\right) \uplus K\left(X_{2}\right)$ (resp. $\left.X=I\left(X_{1}\right) \uplus I\left(X_{2}\right)\right)$ the BCK-bialgebra (resp. BCI-bialgebra). If $\left(X_{1}, *, 0\right)$ is a BCK-algebra (resp. BCI-algebra) and $\left(X_{2}, \oplus, 0\right)$ is a BCI-algebra (resp. BCK-algebra), then we say that $X=(X, *, \oplus, 0)$ is a BCKI-bialgebra (resp. BCIK-bialgebra), and denoted by $X=K\left(X_{1}\right) \uplus I\left(X_{2}\right)$ (resp. $X=I\left(X_{1}\right) \uplus K\left(X_{2}\right)$ ).

Definition 3.2. Let $X=K\left(X_{1}\right) \uplus K\left(X_{2}\right)$ (or $X=K\left(X_{1}\right) \uplus I\left(X_{2}\right), X=I\left(X_{1}\right) \uplus$ $\left.K\left(X_{2}\right), X=I\left(X_{1}\right) \uplus I\left(X_{2}\right)\right)$ be a BCK-bialgebra (or a BCKI-bialgebra, a BCIKbialgerba, a BCI-bialgebra). A subset $H(\neq \emptyset)$ of $X$ is called a biideal of $X$ if there exist distinct proper subsets $H_{1}$ and $H_{2}$ of $X_{1}$ and $X_{2}$, respectively, such that $H=H_{1} \cup H_{2}$ and $H_{i} \triangleleft X_{i}$ for $i=1,2$.

We illustrate this definition by the following examples.

Example 3.3. Let $X=\{0, a, b, c, d, x, y\}$ and consider two proper subsets $X_{1}=$ $\{0, a, b, c, d\}$ and $X_{2}=\{0, a, b, x, y\}$ of $X$ together with Cayley tables respectively 
as follows:

\begin{tabular}{l|lllll}
$*$ & 0 & $a$ & $b$ & $c$ & $d$ \\
\hline 0 & 0 & 0 & 0 & 0 & 0 \\
$a$ & $a$ & 0 & 0 & 0 & 0 \\
$b$ & $b$ & $a$ & 0 & 0 & 0 \\
$c$ & $c$ & $c$ & $c$ & 0 & $c$ \\
$d$ & $d$ & $d$ & $d$ & $d$ & 0
\end{tabular}

\begin{tabular}{l|lllll}
$\oplus$ & 0 & $a$ & $b$ & $x$ & $y$ \\
\hline 0 & 0 & 0 & 0 & 0 & 0 \\
$a$ & $a$ & 0 & 0 & 0 & 0 \\
$b$ & $b$ & $a$ & 0 & 0 & $a$ \\
$x$ & $x$ & $x$ & $x$ & 0 & $x$ \\
$y$ & $y$ & $y$ & $y$ & $y$ & 0
\end{tabular}

Then $X=K\left(X_{1}\right) \uplus K\left(X_{2}\right)$. Note that $H_{1}=\{0, a, b\} \triangleleft X_{1}$ and $H_{2}=\{0, a, b, y\} \triangleleft X_{2}$. Hence $H=\{0, a, b, y\}$ is a biideal of $X$.

Example 3.4. Let $X=\{0, a, b, c, x\}$ and consider two proper subsets $X_{1}=$ $\{0, a, b, c\}$ and $X_{2}=\{0, a, x\}$ of $X$ together with Cayley tables respectively as follows:

\begin{tabular}{c|ccccc|ccc}
$*$ & 0 & $a$ & $b$ & $c$ & $\oplus$ & 0 & $a$ & $x$ \\
\hline 0 & 0 & 0 & 0 & 0 & 0 & 0 & 0 & $x$ \\
$a$ & $a$ & 0 & 0 & $a$ & $a$ & $a$ & 0 & $x$ \\
$b$ & $b$ & $a$ & 0 & $b$ & $x$ & $x$ & $x$ & 0 \\
$c$ & $c$ & $c$ & $c$ & 0 & & & &
\end{tabular}

Then $X=K\left(X_{1}\right) \uplus I\left(X_{2}\right)$, and $I_{1}=\{0, c\} \triangleleft X_{1}$ and $I_{2}=\{0, a\} \triangleleft X_{2}$. Therefore $I=\{0, a, c\}$ is a biideal of $X$.

Example 3.5. Let $X=\{0, a, b, c, d, e, f, g, x, y\}$ and consider two proper subsets $X_{1}=\{0, a, b, c, d, e, f, g\}$ and $X_{2}=\{0, a, x, y\}$ of $X$ together with Cayley tables respectively as follows:

\begin{tabular}{|c|c|c|c|c|c|c|c|c|c|c|c|c|c|}
\hline$*$ & 0 & $a$ & $b$ & $c$ & $d$ & $e$ & $f$ & $g$ & & & & & \\
\hline 0 & 0 & 0 & 0 & 0 & $d$ & $d$ & $d$ & $d$ & & & & & \\
\hline$a$ & $a$ & 0 & 0 & 0 & $e$ & $d$ & $d$ & $d$ & $\oplus$ & 0 & $a$ & $x$ & $y$ \\
\hline$b$ & $b$ & $b$ & 0 & 0 & $f$ & $f$ & $d$ & $d$ & 0 & 0 & 0 & $x$ & $x$ \\
\hline$c$ & $c$ & $b$ & $a$ & 0 & $g$ & $f$ & $e$ & $d$ & $a$ & $a$ & 0 & $x$ & $x$ \\
\hline$d$ & $d$ & $d$ & $d$ & $d$ & 0 & 0 & 0 & 0 & $x$ & $x$ & $x$ & 0 & 0 \\
\hline$e$ & $e$ & $d$ & $d$ & $d$ & $a$ & 0 & 0 & 0 & $y$ & $y$ & $x$ & $a$ & 0 \\
\hline$f$ & $f$ & $f$ & $d$ & $d$ & $b$ & $b$ & 0 & 0 & & & & & \\
\hline$g$ & $g$ & $f$ & $e$ & $d$ & $c$ & $b$ & $a$ & 0 & & & & & \\
\hline
\end{tabular}

Then $X=I\left(X_{1}\right) \uplus I\left(X_{2}\right)$, and $I_{1}=\{0, d\} \triangleleft X_{1}$ and $I_{2}=\{0, a\} \triangleleft X_{2}$. Therefore $I=\{0, a, d\}$ is a biideal of $X$. Note that $I=\{0, a, d\}$ is not an ideal of $\left(X_{1}, *, 0\right)$ since $e * d=a \in I$ and $e \notin I$.

Example 3.6. Let $X=\mathbb{Q}^{*} \cup X_{2}$, where $\mathbb{Q}^{*}$ is the set of all nonzero rational numbers and $X_{2}$ is a BCK-algebra under the operation $\oplus$ that satisfies the following 
implication:

$$
\left(\forall x, y, z \in X_{2}\right)(x \oplus y \leq z, y \leq z \Rightarrow x \leq z) .
$$

Note that $\left(\mathbb{Q}^{*}, \div, 1\right)$ is a BCI-algebra. Thus $X=I\left(\mathbb{Q}^{*}\right) \uplus K\left(X_{2}\right)$. Let $J=A(a) \cup \mathbb{Z}^{*}$, where $\mathbb{Z}^{*}=\mathbb{Z} \backslash\{0\}$ and $A(a)=\left\{x \in X_{2} \mid x \leq a\right\}$ for a fixed element $a$ of $X_{2}$. Then $A(a)$ and $\mathbb{Z}^{*}$ are ideals of $X_{2}$ and $\mathbb{Q}^{*}$, respectively. Hence $J$ is a biideal of $X$.

We provide conditions for a subset to be a biideal.

Theorem 3.7. Let $X=K\left(X_{1}\right) \uplus K\left(X_{2}\right)$ (resp. $X=K\left(X_{1}\right) \uplus I\left(X_{2}\right), X=$ $\left.I\left(X_{1}\right) \uplus K\left(X_{2}\right), X=I\left(X_{1}\right) \uplus I\left(X_{2}\right)\right)$. If $A$ is a nonempty subset of $X$ such that $A \cap X_{1} \triangleleft\left(X_{1}, *, 0\right)$ and $A \cap X_{2} \triangleleft\left(X_{2}, \oplus, 0\right)$, then $A$ is a biideal of $X$.

Proof. It is sufficient to show that $\left(A \cap X_{1}\right) \cup\left(A \cap X_{2}\right)=A$. Now,

$$
\begin{aligned}
\left(A \cap X_{1}\right) \cup\left(A \cap X_{2}\right) & =\left(\left(A \cap X_{1}\right) \cup A\right) \cap\left(\left(A \cap X_{1}\right) \cup X_{2}\right) \\
& =\left(A \cap\left(X_{1} \cup A\right)\right) \cap\left(\left(A \cup X_{2}\right) \cap X\right) \\
& =A \cap\left(A \cup X_{2}\right) \\
& =A .
\end{aligned}
$$

Hence $A$ is a biideal of $X$.

Definition 3.8 ([2]). Let $X=K\left(X_{1}\right) \uplus K\left(X_{2}\right)$ (resp. $X=K\left(X_{1}\right) \uplus I\left(X_{2}\right)$, $\left.X=I\left(X_{1}\right) \uplus K\left(X_{2}\right), X=I\left(X_{1}\right) \uplus I\left(X_{2}\right)\right)$. A subset $H(\neq \emptyset)$ of $X$ is called a sub-bialgebra of $X$ if there exist subsets $H_{1}$ and $H_{2}$ of $X_{1}$ and $X_{2}$, respectively, such that

(i) $H_{1} \neq H_{2}$ and $H=H_{1} \cup H_{2}$,

(ii) $\left(H_{1}, *, 0\right)$ is a subalgebra of $\left(X_{1}, *, 0\right)$,

(iii) $\left(H_{2}, \oplus, 0\right)$ is a subalgebra of $\left(X_{2}, \oplus, 0\right)$.

Theorem 3.9. Let $X=K\left(X_{1}\right) \uplus K\left(X_{2}\right)$ be a BCK-bialgebra. Then any biideal of $X$ is a sub-bialgebra of $X$.

Proof. Straightforward.

The following example shows that the converse of Theorem 3.9 is not true in general.

Example 3.10. Let $X=\{0, a, b, 1,2,3,4\}$ and consider two proper subsets $X_{1}=$ $\{0, a, b\}$ and $X_{2}=\{0,1,2,3,4\}$ of $X$ together with Cayley tables respectively as follows:

\begin{tabular}{c|ccc}
$*$ & 0 & $a$ & $b$ \\
\hline 0 & 0 & 0 & 0 \\
$a$ & $a$ & 0 & 0 \\
$b$ & $b$ & $a$ & 0
\end{tabular}

\begin{tabular}{c|lllll}
$\oplus$ & 0 & 1 & 2 & 3 & 4 \\
\hline 0 & 0 & 0 & 0 & 0 & 0 \\
1 & 1 & 0 & 0 & 0 & 0 \\
2 & 2 & 1 & 0 & 1 & 0 \\
3 & 3 & 1 & 1 & 0 & 0 \\
4 & 4 & 1 & 1 & 1 & 0
\end{tabular}


Then $X=K\left(X_{1}\right) \uplus K\left(X_{2}\right)$. Note that $S_{1}=\{0, a\}$ and $S_{2}=\{0,1,2,3\}$ are subalgebras of $X_{1}$ and $X_{2}$, respectively. Hence $S=\{0, a, 1,2,3\}$ is a sub-bialgebra of $X$. But $S_{1}$ is not an ideal of $X_{1}$ since $b * a=a \in S_{1}$ and $b \notin S_{1}$. Also, $S_{2}$ is not an ideal of $X_{2}$ because $4 \oplus 2=1 \in S_{2}$ and $4 \notin S_{2}$. Therefore $S$ is not a biideal of $X$.

Example 3.11. Let $X=\{0, a, x, y, 1,2,3,4\}$ and consider two proper subsets $X_{1}=\{0,1,2,3,4\}$ and $X_{2}=\{0, a, x, y\}$ of $X$ together with Cayley tables respectively as follows:

\begin{tabular}{c|ccccc}
$*$ & 0 & 1 & 2 & 3 & 4 \\
\hline 0 & 0 & 0 & 0 & 0 & 0 \\
1 & 1 & 0 & 0 & 1 & 0 \\
2 & 2 & 1 & 0 & 2 & 0 \\
3 & 3 & 3 & 3 & 0 & 3 \\
4 & 4 & 4 & 4 & 4 & 0
\end{tabular}

\begin{tabular}{l|llll}
$\oplus$ & 0 & $a$ & $x$ & $y$ \\
\hline 0 & 0 & 0 & $x$ & $x$ \\
$a$ & $a$ & 0 & $x$ & $x$ \\
$x$ & $x$ & $x$ & 0 & 0 \\
$y$ & $y$ & $x$ & $a$ & 0
\end{tabular}

Then $X=K\left(X_{1}\right) \uplus I\left(X_{2}\right)$. Note that $H_{1}=\{0,1,2\}$ is a subalgebra of $X_{1}$ which is an ideal of $X_{1}$, and $H_{2}=\{0, a, x\}$ is a subalgebra of $X_{2}$ but not an ideal of $X_{2}$ since $y \oplus a=x \in H_{2}$ and $y \notin H_{2}$. Hence $H=\{0,1,2, a, x\}$ is a sub-bialgebra of $X$ which is not a biideal of $X$.

Note that any biideal in a BCK-bialgebra $X=K\left(X_{1}\right) \uplus K\left(X_{2}\right)$ is a sub-bialgebra (see Theorem 3.9). But, in a BCKI-bialgebra $X=K\left(X_{1}\right) \uplus I\left(X_{2}\right)$, any biideal is not a sub-bialgebra in general as seen in the following example.

Example 3.12. In Example 3.6, we know that $\mathbb{Z}^{*}$ is an ideal of $\mathbb{Q}^{*}$, but not a subalgebra. So, we know that any biideal is not a sub-bialgebra in $X=K\left(X_{1}\right) \uplus$ $I\left(X_{2}\right), X=I\left(X_{1}\right) \uplus K\left(X_{2}\right)$, or $X=I\left(X_{1}\right) \uplus I\left(X_{2}\right)$.

Example 3.13. Let $X=Y \cup \mathbb{Z}$, where $Y=\{0, a, b, c, d\}$ is a BCK-algebra with the following Cayley table:

\begin{tabular}{l|lllll}
$*$ & 0 & $a$ & $b$ & $c$ & $d$ \\
\hline 0 & 0 & 0 & 0 & 0 & 0 \\
$a$ & $a$ & 0 & $a$ & 0 & 0 \\
$b$ & $b$ & $b$ & 0 & $b$ & 0 \\
$c$ & $c$ & $c$ & $c$ & 0 & $c$ \\
$d$ & $d$ & $d$ & $d$ & $d$ & 0
\end{tabular}

Note that $(\mathbb{Z},-, 0)$ is a BCI-algebra. Hence $X=K(Y) \uplus I(\mathbb{Z})$. It is easy to show that $G_{1}=\{0, a, c\}$ is an ideal of $Y$ which is also a subalgebra of $Y$, and the set $G_{2}=\{x \in \mathbb{Z} \mid 0 \leq x\}$ is an ideal of $\mathbb{Z}$ which is not a subalgebra. Hence $G:=G_{1} \cup G_{2}$ is a biideal of $X$ which is not a sub-bialgebra of $X$.

Definition 3.14. Let $X=K\left(X_{1}\right) \uplus I\left(X_{2}\right)$ (resp. $X=I\left(X_{1}\right) \uplus K\left(X_{2}\right)$ ). A subset 
$A(\neq \emptyset)$ of $X$ is called an IC-ideal (resp. CI-ideal) of $X$ if there exist distinct proper subsets $A_{1}$ and $A_{2}$ of $X_{1}$ and $X_{2}$, respectively, such that

(i) $A=A_{1} \cup A_{2}$,

(ii) $A_{1} \triangleleft X_{1}$ and $A_{2} \triangleleft_{c} X_{2}$ (resp. $A_{1} \triangleleft_{c} X_{1}$ and $A_{2} \triangleleft X_{2}$ ).

Note that any IC-ideal (resp. CI-ideal) in $X=K\left(X_{1}\right) \uplus I\left(X_{2}\right)$ (resp. $X=$ $\left.I\left(X_{1}\right) \uplus K\left(X_{2}\right)\right)$ is a biideal, but the converse is not true in general.

Example 3.15. (1) In Example 3.13, $G:=G_{1} \cup G_{2}$ is a biideal which is not an IC-ideal since $G_{2}$ is not closed.

(2) In Example 3.5, $I=\{0, a, d\}$ is an IC-ideal of $X$.

Theorem 3.16. Let $X=K\left(X_{1}\right) \uplus I\left(X_{2}\right)$ (resp. $\quad X=I\left(X_{1}\right) \uplus K\left(X_{2}\right)$ ), where $\left|X_{2}\right|<\infty$ (resp. $\left|X_{1}\right|<\infty$ ). Then every biideal of $X$ is an IC-ideal (resp. CIideal) of $X$.

Proof. Assume that $X=K\left(X_{1}\right) \uplus I\left(X_{2}\right)$ and $\left|X_{2}\right|=n<\infty$. Let $A$ be a biideal of $X$. Then there are distinct subsets $A_{1}$ and $A_{2}$ of $X_{1}$ and $X_{2}$, respectively, so that $A=A_{1} \cup A_{2}$ and $A_{i} \triangleleft X_{i}$ for $i=1,2$. For every $a, b \in A_{2}$ and $k \in \mathbb{N}$, denote

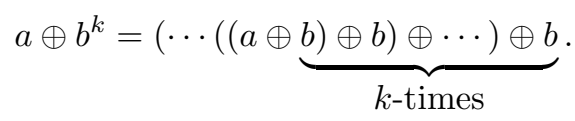

Now, for each $a \in A_{2}$, consider $n+1$ elements as follows:

$$
0,0 \oplus a, 0 \oplus a^{2}, \cdots, 0 \oplus a^{n} .
$$

Since $\left|X_{2}\right|=n$, it follows that two of them must be equal so that there exist $r, s \in \mathbb{N}$ such that $s<r \leq n$ and $0 \oplus a^{r}=0 \oplus a^{s}$. Then

$$
0=\left(0 \oplus a^{r}\right) \oplus\left(0 \oplus a^{s}\right)=\left(\left(0 \oplus a^{s}\right) \oplus a^{r-s}\right) \oplus\left(0 \oplus a^{s}\right)=0 \oplus a^{r-s} \in A_{2},
$$

and so $0 \oplus a \in A_{2}$ since $A_{2} \triangleleft X_{2}$. Thus $A_{2} \triangleleft_{c} X_{2}$. Therefore $A$ is an IC-ideal of $X$. Similarly we get desired result for the case $X=I\left(X_{1}\right) \uplus K\left(X_{2}\right)$ with $\left|X_{1}\right|<\infty$.

Corollary 3.17. Let $X=I\left(X_{1}\right) \uplus I\left(X_{2}\right)$, where $\left|X_{1}\right|<\infty$ and $\left|X_{2}\right|<\infty$. Then every biideal of $X$ is a $C C$-ideal of $X$.

Theorem 3.18. Let $X=K\left(X_{1}\right) \uplus I\left(X_{2}\right)$ (resp. $X=I\left(X_{1}\right) \uplus K\left(X_{2}\right)$ ). Then any IC-ideal (resp. CI-ideal) of $X$ is a sub-bialgebra of $X$.

Proof. It is straightforward because any closed ideal of a BCI-algebra is a subalgerba, and any ideal of a BCK-algebra is a subalgebra.

The following example shows that the converse of Theorem 3.18 is not true in general.

Example 3.19. Let $X=\{0, a, b, 1,2,3,4\}$ and consider two proper subsets $X_{1}=$ 
$\{0,1,2,3,4\}$ and $X_{2}=\{0, a, b\}$ of $X$ together with Cayley tables respectively as follows:

\begin{tabular}{c|ccccc}
$*$ & 0 & 1 & 2 & 3 & 4 \\
\hline 0 & 0 & 0 & 0 & 0 & 0 \\
1 & 1 & 0 & 1 & 1 & 1 \\
2 & 2 & 2 & 0 & 2 & 2 \\
3 & 3 & 3 & 3 & 0 & 3 \\
4 & 4 & 4 & 4 & 4 & 0
\end{tabular}

\begin{tabular}{c|ccc}
$\oplus$ & 0 & $a$ & $b$ \\
\hline 0 & 0 & 0 & $b$ \\
$a$ & $a$ & 0 & $b$ \\
$b$ & $b$ & $b$ & 0
\end{tabular}

Then $X=K\left(X_{1}\right) \uplus I\left(X_{2}\right)$, and $H_{1}=\{0,1,2,4\}$ is a subalgebra of $X$ which is also an ideal. But $H_{2}=\{0, b\}$ is a subalgebra of $X_{2}$ which is not an ideal of $X_{2}$. Hence $H:=\{0,1,2,4, b\}$ is a sub-bialgebra of $X$ which is not an IC-ideal.

Corollary 3.20. Let $X=K\left(X_{1}\right) \uplus I\left(X_{2}\right)$ (resp. $\quad X=I\left(X_{1}\right) \uplus K\left(X_{2}\right)$ ), where $\left|X_{2}\right|<\infty$ (resp. $\left.\left|X_{1}\right|<\infty\right)$. Then every biideal of $X$ is a sub-bialgebra of $X$.

Theorem 3.21. Let $X=K\left(X_{1}\right) \uplus I\left(X_{2}\right)$ in which $X_{2}$ satisfies the following inequality:

$$
\left(\forall x \in X_{2}\right)(0 \oplus x \leq x) .
$$

Then any biideal of $X$ is an IC-ideal of $X$ and hence is a sub-bialgebra of $X$.

Proof. Let $A$ be a biideal of $X$. Then $A=A_{1} \uplus A_{2}$ and $A_{i} \triangleleft X_{i}, i=1,2$, for some $A_{1} \subseteq X_{1}$ and $A_{2} \subseteq X_{2}$ with $A_{1} \neq A_{2}$. Let $y \in A_{2}$. Since $0 \oplus y \leq y$ by assumption, it follows that $0 \oplus y \in A_{2}$ so that $A_{2} \triangleleft_{c} X_{2}$. Hence $A$ is an IC-ideal of $X$.

Theorem 3.22. Let $X=K\left(X_{1}\right) \uplus I\left(X_{2}\right)$ (resp. $X=I\left(X_{1}\right) \uplus K\left(X_{2}\right)$ ) and let $A$ be a subset of $X$ such that $A \cap X_{1} \triangleleft\left(X_{1}, *, 0\right)$ and $A \cap X_{2} \triangleleft_{c}\left(X_{2}, \oplus, 0\right)$ (resp. $A \cap X_{1} \triangleleft_{c}\left(X_{1}, *, 0\right)$ and $\left.A \cap X_{2} \triangleleft\left(X_{2}, \oplus, 0\right)\right)$. Then $A$ is an IC-ideal (resp. CI-ideal) of $X$.

Proof. Similar to the proof of Theorem 3.7.

Acknowledgment. The author is highly grateful to referees for their valuable comments and suggestions helpful in improving this paper.

\section{References}

[1] K. Iséki and S. Tanaka, An introduction to the theory of BCK-algebras, Math. Japonica, 23(1)(1978), 1-26.

[2] Y. B. Jun, M. A. Öztürk and E. H. Roh, BCK/BCI-bialgebras, Sci. Math. Jpn., 64(3)(2006), 595-600, e2006, 903-908.

[3] J. Meng and Y. B. Jun, BCK-algebras, Kyungmoon Sa Co., Seoul, 1994. 
[4] W. B. Vasantha Kandasamy, Bialgebraic structures and Smarandache bialgebraic structures, American Research Press, 2003.

(http://www.gallup.unm.edu/ smarandache/eBooks-otherformats.htm) 